Results: Demographics, disease characteristics and nailfold video-capillaroscopic (NVC) pattern details were summarised in table-1. The scores of EUSTAR and EScSG activity indexes were correlated well $(r=0.576$, $\mathrm{p}=0.000$ ) and the agreement between two scores for activity was moderate (cohen kappa:0.407). The percentages of SSc patients described as having active disease or not according to two activity indexes were summarised in table-2. Of the patients, 9.9\% had active and $70.9 \%$ had inactive disease according to both indexes in this SSc cohort. Twenty one (for EUSTAR) and 4 patients (for EScSG) were described as active according to one index and not to the other. Revised EUSTAR activity index was found to have $76.5 \%$ sensitivity and $81.6 \%$ specificity when the activity was defined by EScSG activity index.

Table 1.

Table-1: Demogranhics, Characteristics and NVC pattem in

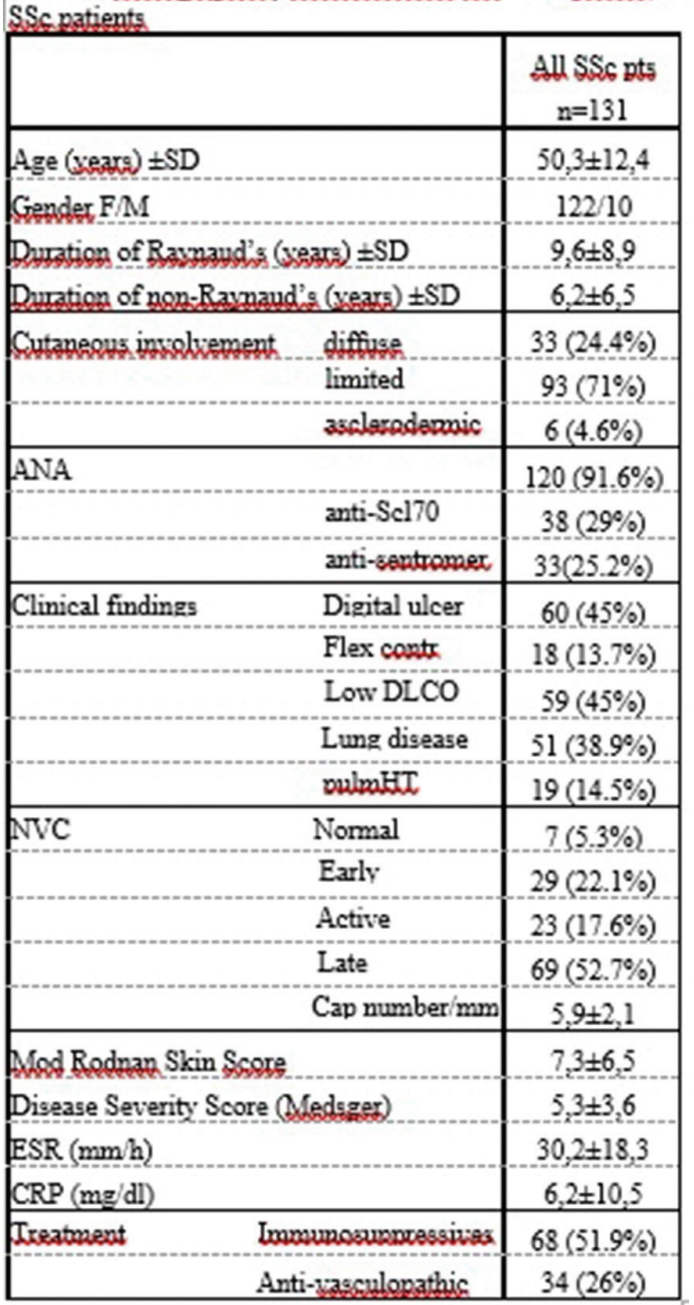

Table 2

Table-2: The Bercentage of the Batients as haxing active or inactive disease acserding to indexes

\begin{tabular}{|c|c|c|c|}
\hline & & \multicolumn{2}{|c|}{ EUSTAR activity index } \\
\hline & & active & inactive \\
\hline \multirow{2}{*}{ ESCSSG actixity index } & active & $13(9.9 \%)$ & $4(3 \%)$ \\
\cline { 2 - 4 } & inactive & $21(16 \%)$ & $93(70.9 \%)$ \\
\hline
\end{tabular}

Conclusion: This SSc cohort predominantly had limited cutanous disease, digital vasculopathy and late scleroderma pattern. Defining active disease was differed in $19 \%$ of the patients according to EUSTAR and EScSG activity indexes, former described higher frequency for activity. This difference might be related to validation procedures of these indexes in patients with different predominant stages of SSc disease, content of the index and the features of the cohort.

Disclosure of Interests: None declared

DOI: 10.1136/annrheumdis-2019-eular.6167

\section{AB0224 \\ SYSTEMIC SCLEROSIS SINE SCLERODERMA: CHALLENGES WITH VERIFICATION IN PATIENTS WITH PULMONARY ARTERIAL HYPERTENSION}

Natalia Yudkina, Alexander Volkov, Ekaterina Nikolaeva, Ildar Kurmukov, Evgeny Nasonov. V.A.Nasonova Research Institute of Rheumatology, Moscow, Russian Federation

Background: Recognizing systemic sclerosis (SSc) in patients without cutaneous involvement is a real challenge due to inapparent clinical picture. 1980 ACR classification criteria for SSc fail to verify the diagnosis in the majority of cases. Therefore, the diagnosis is usually established years and decades after SSc onset at the stage of full-blown visceral disease. Introduction of 2013 ACR/EULAR classification criteria into clinical practice allow early diagnosis of SSc even in cases without skin involvement thanks to the fact, that list of considered criteria includes telangiectasia, pulmonary arterial hypertension (PAH), abnormal nailfold capillaries and SSc-related autoantibodies.

Objectives: To identify specific features in the clinical course of SSc sine scleroderma (ssSSc) in patients with PAH

Methods: 11 patients with verified SSc diagnosis according to 2013 criteria were included; participants did not have any SSc-specific signs of skin involvement, such as puffy fingers, fingers' skin thickening/induration or skin atrophy. PAH was diagnosed in 11 patients during of right heart catheterization.

Results: Isolated Raynaud's phenomenon (RP) was along-standing diagnosis (more than 5 years) in all patients, except for one whoman, in whom the disease manifested with signs of Sjogren's syndrome (SjS) (parotitis). Benign, chronic, and gradually progressing during a long time disease was documented in all patients. In 7 patients out of 11 SSc diagnosis was initially suspected by cardiologists, and later confirmed by detection of antinuclear autoantibodies. The following signs were documented in SSc patients as initial manifesting non-Raynaud symptoms: esophageal dysmotility - in 3 patients, digital ulcers - in 2, telangiectasia - in 2 patients, and dyspnea (PAH symptom) - in 1 patient. None of the patients ever experienced skin thickening typical for SSc. RP was present in all $100 \%$, but digital ischemic alterations - digital tip ulcers, pitting scars were found only in 3 patients. SSc diagnosis was verified based on abnormal nailfold capillaries (in 100\% cases), and based on identification of SSc-specific antibodies (anticentromere antibodies - ACA) - in 7 patients. Anti-Ro-antibodies were found in 4 patients, anti-RNP-70 - in 3 SjS was established in 3 out of 4 anti-Ro-antibodies - positive patients and ruled out in one. Myositis was documented in past-medical history in one patient with anti-RNP-70-antibodies positivity. It should be noted, that 3 ACA-negative patients had, nevertheless, a "threshold" score value (9 scores) for SSc diagnosis, exhibiting many clinical features in favor of SSc diagnosis beyond any doubt. Esophageal dysmotility and associated symptoms were found in 10 out of 11 SSc patients. One patient had an SSc-rheumatoid arthritis overlap syndrome (erosive arthritis, joint deformities, positive ACA, rheumatoid factor, anti-CCP).

Conclusion: Introduction of 2013 ACR-EULAR classification criteria for SSc into clinical practice was highly relevant for identification of SSc cases without cutaneous involvement, providing therefore a timely diagnosis in this group of patients, and allowing to prognosticate the clinical course of PAH. 2013 ACR-EULAR classification criteria for SSc is an algorithm for SSc verification in patents with $\mathrm{PAH}$, especially in the absence of typical for SSc cutaneous disease. The earlier PAH-SSc is diagnosed and $\mathrm{PAH}$-specific therapy is initiated, the longer will be patient' lifespan.

Disclosure of Interests: Natalia Yudkina: None declared, Alexander Volkov : None declared, Ekaterina Nikolaeva: None declared, Ildar Kurmukov: None declared, Evgeny Nasonov Speakers bureau: Pfizer, Inc. MSD, Novartis, AbbVie Inc., Celgen Corporation, Biocad, Janssen, UCB Inc.

DOI: 10.1136/annrheumdis-2019-eular.7207

\section{$\mathrm{AB} 0225$ \\ CLINICALSUBTYPE OF PULMONARY ARTERIAL HYPERTENSION ASSOCIATED WITH SYSTEMIC SCLEROSIS}

Natalia Yudkina, Alexander Volkov, Ekaterina Nikolaeva, Ildar Kurmukov, Evgeny Nasonov. V.A.Nasonova Research Institute of Rheumatology, Moscow, Russian Federation

Background: Despite the similar pathogenesis and clinical picture, pulmonary arterial hypertension in systemic sclerosis (PAH-SSc) in comparison with idiopathic pulmonary arterial hypertension (IPAH) is characterized by a more severe course, an unsatisfactory response to PAH-specific therapy, a poor survival and a 